\title{
A Preliminary Study on Land Use Effects on Birds Diversity in the Selected Agro-Ecosystems of Ekiti State, Nigeria
}

\section{Ogunyemi 00*}

Department of Forest Resources and Wildlife Management, Ekiti State University, Nigeria

*Corresponding author: Ogunyemi 00, Department of Forest Resources and Wildlife Management, Ekiti State University, Ado-Ekiti, Nigeria, Email: olumideogunyemi80@yahoo. com

\section{Research Article}

Volume 4 Issue 5

Received Date: August 24, 2020

Published Date: September 17, 2020

DOI: $10.23880 /$ jenr- 16000212

\section{Abstract}

Birds as bio-indicator of an environment play an important role in the maintenance of balance in an ecosystem by providing various ecological services. The land-use effects on bird species diversity in Ekiti State were investigated to determine and compare the diversity, guild, structure and relative abundance in the four selected land use types of Ekiti State. Four study areas were selected among the land use types in the state. Transect count technique was employed to collect data on birds diversity and abundance. A total of 392 individual birds were observed that included 85 bird species belonging to 14 orders and 35 families. The order Passeriformes constituted the numerically dominant order represented with 37 species which represents $43.5 \%$ of the recorded bird species. Among the land use types, the highest species diversity was found in the natural forest $\left(\mathrm{H}^{\prime}=3.34\right)$ and the lowest was recorded in cashew plantation (2.88). There exist significant difference in the abundance of bird species and the total birds sighted in the four land use types at $5 \%$ level of significance $(F=2.09, P=6.33)$. The bird species composition of cashew plantation was more similar to that of cocoa plantation. The land-use features of the study areas and the resources abundance proved vital to the diversity of birds in the land uses as evidenced by the high species richness and abundance of insectivorous species in the study areas. Therefore, this study provide evidence that land-use types as a form of habitat modification may alter bird diversity structure and that the maintenance of the land use features will assist in the conservation of the birds in Ekiti State.

Keywords: Diversity; Guild; Structure; Relative Abundance

\section{Introduction}

The significance of land use type near wildlife habitats has been recognized globally as the major drive of biodiversity loss [1-3]. Roughly one- third of the global land area is devoted to cultivated land use system and as a consequence more area is expected to be converted to agricultural land use practices. Conservation in the tropics has its focus on pristine habitats such as rainforest [4], due to high level of biodiversity presence whereas human dominated landscape have been all but ignored in tropical ecological and conservation research as they support lower species diversity [5]. Over the last 30 years the natural habitats has been dwindling in area, which necessitates much of wildlife relocating and relies on land heavily used by humans and that the types and scale of land use types can have a marked influence on the wildlife populations' structure [6,7]. Consequent upon the spreading of agricultural landscapes decreasing at an alarming rate and pristine habitats shrinking, survival of many biodiversity invariably become the function of the types and patterns of land use which must be understood in order to incorporate these lands into conservation management plans. Land use can be a major source of feeding and breeding site for birds and a temporary or permanent habitat for some species [8]. Land use for agricultural purposes such as shade-grown cocoa was reported to harbour high biodiversity due to the presence of diverse high canopy forming species, complex forest structure, and absence of invasive exotic weeds [9]. 


\section{Journal of Ecology and Natural Resources}

Such land use type often provides suitable habitats for native fauna and were especially good for birds. In contrast, sun-grown monocultures plantation of Cocoa, coffee and oil palm were found to have adverse effects on biodiversity due to homogeneity and presence of invasive weed species [10]. Most deforestation has taken place in biodiversity rich tropical forests, these areas are expected to face even more pressures in the future, due to agricultural expansion. Conversion of natural habitats, especially intact old-growth forest to agricultural and pastoral lands is among the greatest threats to biodiversity in the recent times. Land use and its management have a major impact on natural resources including water, soil, nutrients, plants and animals and tend to vary from one country to another [11]. Land use could result into complete transformation, such as transforming a forest land to the settlement land or it could involve partial transformation through retaining the primary status of the land such as vegetation covers [12]. In most tropical forest areas, agricultural lands are predominant types of land use around native forest patches [13], but their influence alone on the persistence of fauna diversity in some tropical regions is poorly understood. Agricultural lands can be essential components in biodiversity conservation within tropical forest-agricultural landscapes if properly managed [14]. The isolation of protected areas as the sole means of protecting biodiversity is insufficient given the current trend in land use dynamics [9]. Many studies have demonstrated the potentials of diverse land uses in supporting biological diversity and stress their integration in conservation strategies [15]. Land uses when properly managed to an extent will not only support a large number of biodiversity but also serve as safe corridors that will permit dispersal of wildlife between patches [16]. Perfecto and Vandermeer [17] proposed that managed agricultural areas were equally important as the forest patches they surround. In their study, they found that species richness of ground-foraging ants in a well shaded organic Cocoa farm did not differ from that of a nearby Montane forest. Similarly, Harvey, et al. [18] confirmed the conservation value of agricultural lands, mainly areas that retained an abundant native tree cover. Such areas as suggested, often exhibited structural heterogeneity while providing habitat and resources for native fauna species [19]. It is known that tree and bird species are most sensitive to change and hence the most critical indicators of the biodiversity impact of land use conversion [20]. Ekiti state is among the areas that have severe land transformation due to various forms of human economic activities. These have resulted to intensive degradation that has transformed most of the natural environment, which in turn influence rate of biodiversity loss [21]. One of the pertinent challenges with which nature conservationist will be confronted within the nearest future will obviously be the discovering the relationship that exist between the changing environments and how land uses as the major driving force in the alteration of pristine ecosystems can be managed effectively to accommodate both human needs and biodiversity conservation. Hence there is a great need to establish the contribution of various forms of land uses on the sustainability of biodiversity.

\section{Methodology}

\section{Study area}

The study was carried out in Ekiti state which lies within the tropics and located between longitude $4^{\circ} 55^{\prime}$ to $5^{\circ} 45^{\prime}$ East of Greenwich meridian and latitude $7^{\circ} 15^{\prime}$ to $8^{\circ}$ $15^{\prime}$ north of the equator. The prevailing climate of the state is tropical, with average temperature of $25^{\circ} \mathrm{C}$ all year round and high relative humidity. The rainy season has an average of 270 days with mean annual rainfall of 1250 to 1400 $\mathrm{mm}$. The pattern of rainfall distribution over the long rainy season between April and mid- November is bimodal with a peak in September while the dry season stretches from mid-November to the end of March. The vegetation of the state is diverse and has been described by several authors to fall in to forest eco-climate vegetation. The study area is characterized by secondary forest and three types of land use practices which are Cashew, Cocoa, Gliricidia plantations and Natural forest designated T1, T2, T3, and T4 respectively. The three study sites were selected from the prominent plantations in Ekiti State. (Site T1): Cashew plantation has from $5 \mathrm{~km}$ (length) to $7.5 \mathrm{~km}$ (width) and is characterized by shrub heights cashew trees and tall cashew trees $(5-7 \mathrm{~m})$ with dense canopy. (Site T2): Cocoa plantation ranges from $8 \mathrm{~km}$ (length) to $6.5 \mathrm{~km}$ (width) and is characterized by high frequency of fairly tall trees $(6-7 \mathrm{~m})$ with a moderately dense canopy. (Site T3): Gliricidia plantation ranges from $5 \mathrm{~km}$ (length) to 6km (width) and has fewer tall trees (6.5- 7m) with a sparse canopy while (Site 4): Natural secondary forest ranges from $6 \mathrm{~km}$ (Length) to $8 \mathrm{~km}$ (width) and characterized high diversity of tree species with high frequency of both fairly tall and tall trees that ranges between 7-12.3m with dense canopy.

\section{Data Collection}

Survey of distribution and diversity of bird species was carried out in each of the four selected land use types (Natural forest, Cocoa, Gliricidia and Cashew plantations) for three consecutive months (April - June, 2019). Fixed length transect count method was employed for the assessment as described by Bibby, et al. [22]. The relative value of each land use types for accommodating bird species was determined by establishment of 3 transects of $1 / 2$ kilometer length in each of the selected four land use types. The birds were observed by 


\section{Journal of Ecology and Natural Resources}

walking along the established transects with the assistance of observers for four consecutive days in a month for the duration of 3 months. Data collection commenced about 30 minutes after dawn from $6.30-9.30 \mathrm{am}$ in the morning and $4.30-6.30 \mathrm{pm}$ in the evening to coincide with time the activities of birds was prominent [23]. To avoid the effects of pseudo-replication in the counting of birds, transect was reasonably space out by $300 \mathrm{~m}$ distance apart [24].

In all transects, record was made of all the types and group number of bird species and food habits through direct observation. The birds were observed by naked eyes and with the aid of binoculars where visibility was low and their taxonomic groups were categorized base on field guide to birds of Western Africa [25].

\section{Data Analysis}

The data collected from the land use types were arranged, organized and entered into Microsoft excel spread sheet for analysis. Shannon-Weiner diversity index $\left(\mathrm{H}^{\prime}\right)$ was used to analyze bird diversity of the land use. It was calculated as

$$
\mathrm{H}^{\prime}=-\sum_{(\mathrm{ni} / \mathrm{N})} \mathrm{In}(\mathrm{ni} / \mathrm{N})
$$

Where: $\mathrm{H}^{\prime}=$ index of species diversity, ni is the number of individual in species, $\mathrm{S}$ is the total number of species, $\mathrm{N}$ is the total number of individuals.

Evenness index (J') was calculated by following the equation.

$$
\mathrm{J}^{\prime}=\mathrm{H}^{\prime} / \mathrm{InS}
$$

Where: $\mathrm{H}=$ Shannon Weiner diversity index and $\mathrm{S}=$ Number of species

Richness index (D) was calculated by the following equation

$$
\mathrm{D}=\mathrm{S}-1 / \mathrm{InN}
$$

Where $\mathrm{D}=$ Richness index, $\mathrm{S}=$ total number of species and $\mathrm{N}=$ total number of individuals

Relative abundance of bird species was determined using encounter rates that give basic ordinal scales of abundance (abundant, common, frequent, uncommon and rare) [23]. Encounter rate of each species was calculated by dividing the number of birds observe by the number of hours spent searching, giving a figure of birds per hour or each species. The categories were: <0.1, 0.1-2.0, 2.1-10.0, 10.1-40.0 and $>40$. For each category, the following abundance score was given: 1(Rare), 2(Uncommon), 3(frequent), 4(common), 5(abundant) respectively. Moreover to understand bird community similarity among sites, Sorensen's coefficient was applied. It was calculated as:

$$
S=3 c / A+B+C+D
$$

Where S=Sorenson's Coefficient, $\mathrm{c}$ is the number of species the four land use types have in common, $\mathrm{A}$ is the total number of bird species found in land use type A, B is the total number of bird species found in land use type $B$ and $C$ is the total number of bird species found in the land use type $C$ while $D$ is the number of species in land use type D. Sorenson's coefficient gives a value between 0 and 1 , the closer the value is to 1 , the more the land use types have in common. To check the presence of variation in bird's parameters among the four study areas One Way ANOVA was used to determine whether there was a significant difference between land uses in term of birds' abundance, richness and diversity.

\section{Results}

\section{Bird Species Richness in the Selected Four Land Use Types of Ekiti State}

A total of 85 bird species were identified belonging to 14 orders and 35 families. The order Passeriformes constituted the numerically dominant order represented with 37 species which represents $43.5 \%(n=37)$ of the recorded species while Psittaciformes, Musophagiformes, Ciconiiformes, Apodiformes and Pelecaniformes were the least dominant orders with $7.14 \%, 14.29 \%, 7.14 \%$, $14.29 \%, 14.29 \%$ respectively (Table 1 ). At the family level, family nectariniidae ranked numerically highest represented by 9 species which accounts $10.59 \%$ of the identified species (Table 1). At species level Laughing Dove (Streptopelia senegalensis), Variable Sunbird (Cinnyris venustus), Red Eye Dove (Streptopelia semitorquata), Red Chested Cuckoo (Cuculux solitaries), Olive-billed Sunbird (Nectarinia chloropygium), Green headed sunbird (Nectarinia vaticalis), L ittle Swift ( Apus affinis) and Copper Sunbird (Cinnyris cupreus) were numerically the dominant species with 16,15 , $14,13,12,12,12$ and12 number of individual respectively given rise to $27.11 \%$ of the total birds abundance observed in the study land use types from the result, it was gathered that the natural forest had the highest species richness compared to the other three (3) land use while the cashew plantation (T1) had the lowest bird species richness value. The natural forest had the lowest individual birds' abundance but it had the highest bird species richness while gliricidia plantation (T3) recorded lower species richness but had the highest number of individual birds (Table 2). 
Journal of Ecology and Natural Resources

\begin{tabular}{|c|c|c|c|c|c|c|c|c|}
\hline$S / N$ & $\begin{array}{c}\text { Common and Scientific } \\
\text { Name }\end{array}$ & Family & Order & T1 & $\mathrm{T} 2$ & T3 & T4 & Total \\
\hline 1. & $\begin{array}{c}\text { White-Rumped Seed } \\
\text { Eatercrithagra leucopygia }\end{array}$ & Fringillidae & Passeriformes & 4 & - & - & - & 4 \\
\hline 2. & $\begin{array}{c}\text { Diederik Cuckoo } \\
\text { Chrysococcyx caprius }\end{array}$ & Cuculidae & Cuculiformes & 8 & - & - & - & 8 \\
\hline 3. & $\begin{array}{l}\text { Common Kestrel } \\
\text { Falco tinnunculus }\end{array}$ & Falconidae & Falconiformes & 2 & - & - & - & 2 \\
\hline 4. & $\begin{array}{c}\text { Piping Hornbill Bycanistes } \\
\text { fistulator }\end{array}$ & Bucerotidae & Bucerotiformes & 2 & - & - & 1 & 3 \\
\hline 5. & $\begin{array}{c}\text { Senegal Coucal } \\
\text { Centropus senegalensis }\end{array}$ & Cuculidae & Cuculiformes & 3 & - & 1 & - & 4 \\
\hline 6. & $\begin{array}{c}\text { Little Bittern } \\
\text { Arderailus sturmii }\end{array}$ & Ardeidae & Pelecaniformes & 5 & - & - & 2 & 7 \\
\hline 7. & $\begin{array}{l}\text { Yellow Billed Shrike } \\
\text { Corvinella corvine }\end{array}$ & Laniidae & Piciformes & 2 & - & - & - & 2 \\
\hline 8. & $\begin{array}{c}\text { Olive-bellied Sunbird } \\
\text { Nectarinia chloropygium }\end{array}$ & Nectarinidae & Passeriformes & 12 & - & - & - & 12 \\
\hline 9. & $\begin{array}{l}\text { Black Shouldered Kite } \\
\text { Elanus caeruleus }\end{array}$ & Accipitridae & Falconiformes & 3 & - & - & - & 3 \\
\hline 10. & $\begin{array}{c}\text { Grey throated barbet } \\
\text { Gymnobucco bonaparter }\end{array}$ & Capitonidae & Piciformes & 2 & 3 & - & - & 5 \\
\hline 11. & $\begin{array}{l}\text { Green headed sunbird } \\
\text { Nectarinia vaticalis }\end{array}$ & Nectarinidae & Passeriformes & 1 & - & 11 & - & 12 \\
\hline 12. & $\begin{array}{l}\text { African pied wagtail } \\
\text { Motacilla aguimp }\end{array}$ & Motacillidae & Passeriformes & - & - & 3 & - & 3 \\
\hline 13. & $\begin{array}{l}\text { Red-headed Weaver } \\
\text { Ploceus cucullatus }\end{array}$ & Ploceidae & Passeriformes & 4 & - & - & - & 4 \\
\hline 14. & $\begin{array}{l}\text { Red Chested Cuckoo } \\
\text { Cuculux solitaries }\end{array}$ & Cuculidae & Cuculiformes & 5 & 8 & - & - & 13 \\
\hline 15. & $\begin{array}{c}\text { Slender-billed Weaver Ploceus } \\
\text { pelzelni }\end{array}$ & Ploceidae & Passeriformes & 4 & 2 & - & - & 6 \\
\hline 16. & $\begin{array}{l}\text { Straw Tailed whydah } \\
\text { Vidua fischeri }\end{array}$ & Motacillidae & Passeriformes & 2 & - & - & - & 2 \\
\hline 17. & $\begin{array}{l}\text { Swallow Tailed Kite } \\
\text { Chelictinia riocourii }\end{array}$ & Accipitridae & Falconiformes & 1 & 1 & - & - & 2 \\
\hline 18. & $\begin{array}{l}\text { Golden backed weaver } \\
\text { Ploceus jacaksoni }\end{array}$ & Ploceidae & Passeriformes & 2 & - & - & - & 2 \\
\hline 19. & Tawny pipit Anthus campestris & Motacillidae & Passeriformes & 2 & - & 2 & - & 4 \\
\hline 20. & $\begin{array}{c}\text { Emerald Cuckoo } \\
\text { Chrysococcyx cupreus }\end{array}$ & Cuculidae & Cuculiformes & 1 & 3 & - & 4 & 8 \\
\hline 21. & $\begin{array}{c}\text { Fork-Tailed Drongo } \\
\text { Dicrurus adsimilis }\end{array}$ & Dicruridae & Passeriformes & - & - & - & 1 & 1 \\
\hline 22. & $\begin{array}{l}\text { Green bee-eater } \\
\text { Merops orientalis }\end{array}$ & Meropidae & Coraciiformes & 8 & - & - & - & 8 \\
\hline
\end{tabular}


Journal of Ecology and Natural Resources

\begin{tabular}{|c|c|c|c|c|c|c|c|c|}
\hline 23. & $\begin{array}{c}\text { Crowned eagle } \\
\text { Stephanoaetus caronatus }\end{array}$ & Accipitridae & Accipitriformes & - & 2 & - & - & 2 \\
\hline 24. & $\begin{array}{l}\text { Wattled black hornbill } \\
\text { Cerotogyina atrata }\end{array}$ & Bucerotidae & Coraciiformes & - & 2 & - & - & 2 \\
\hline 25. & \begin{tabular}{|lr} 
Black Kite & Mulvus \\
& migrans \\
\end{tabular} & Accipitridae & Falconiformes & - & 4 & - & - & 4 \\
\hline 26. & $\begin{array}{l}\text { Crested Guineafowl } \\
\text { Guttera edouardi }\end{array}$ & Numididae & Galliformes & - & 1 & - & - & 1 \\
\hline 27. & $\begin{array}{c}\text { African pipit } \\
\text { Anthus cinnamomeus }\end{array}$ & Motacillidae & Passeriformes & - & 2 & - & - & 2 \\
\hline 28. & $\begin{array}{l}\text { Tawny Eagle } \\
\text { Aquila rapax }\end{array}$ & Accipitridae & Falconiformes & - & 4 & - & - & 4 \\
\hline 29. & $\begin{array}{l}\text { Little bee-eater } \\
\text { Merops pusillus }\end{array}$ & Meropidae & Coraciiformes & - & 2 & - & - & 2 \\
\hline 30. & $\begin{array}{c}\text { Wattle Ibis } \\
\text { Bastrychia carunculata }\end{array}$ & Threskiomithidae & Ciconiiformes & - & 5 & - & - & 5 \\
\hline 31. & $\begin{array}{l}\text { Red- Necked spurfowl } \\
\text { Francolinus afer }\end{array}$ & Numididae & Galliformes & - & 4 & 1 & - & 5 \\
\hline 32. & $\begin{array}{l}\text { Yellow-rumped Tinkerbird } \\
\text { Pogoniulus bilineatus }\end{array}$ & Lybiidae & Piciformes & - & - & - & 3 & - \\
\hline 33. & Little Swift Apus affinis & Apodidae & Apodiformes & - & 10 & 2 & - & 12 \\
\hline 34. & $\begin{array}{l}\text { White Browned Coucal } \\
\text { Centropus superciliosus }\end{array}$ & Cuculidae & Cuculiformes & - & 3 & - & - & 3 \\
\hline 35. & $\begin{array}{l}\text { Yellow-fronted Tinkerbird } \\
\text { Pogoniulus chrysoconus }\end{array}$ & Lybiidae & Piciformes & - & - & - & 6 & 6 \\
\hline 36. & $\begin{array}{l}\text { Yellow Throated Tinkerbird } \\
\text { Pogoniulus subsulphure }\end{array}$ & Lybiidae & Piciformes & - & - & - & 3 & 3 \\
\hline 37. & $\begin{array}{l}\text { Grey Throated Barbet } \\
\text { Gymnobucco bonaparter }\end{array}$ & Capitonidae & Piciformes & - & - & - & 1 & 1 \\
\hline 38. & $\begin{array}{c}\text { Red Chested Sunbird } \\
\text { Nectarinia erythrocerca }\end{array}$ & Nectariniidae & Passeriformes & - & 2 & - & - & 2 \\
\hline 39. & Pied Crow Corvus capensis & Corvidae & Passeriformes & - & 3 & 7 & - & 10 \\
\hline 40. & $\begin{array}{l}\text { White-tailed Ant Thrush } \\
\text { Neocossyphus poensis }\end{array}$ & Turdidae & Passeriformes & - & - & - & 1 & 1 \\
\hline 41. & $\begin{array}{l}\text { Capped Wheater } \\
\text { Oenanthe pileata }\end{array}$ & Pycronotidae & Passeriformes & - & 1 & - & - & 1 \\
\hline 42. & Dwarf Raven Corvus splendens & Corvidae & Passeriformes & - & 1 & - & - & 1 \\
\hline 43. & Pied cuckoo Clamator jacobinus & Cuculidae & Cuculiformes & - & - & - & 2 & 2 \\
\hline 44. & $\begin{array}{l}\text { Black And White Casqued } \\
\text { Hornbill Byconistes bucinator }\end{array}$ & Bucerotidae & Coraciformes & - & 6 & - & - & 6 \\
\hline 45. & African Darter Anhinga rufa & Phalacrocoracidae & Pelecaniformes & - & 4 & - & - & 4 \\
\hline 46. & $\begin{array}{c}\text { Red Bellied Paradise Flycatcher } \\
\text { Terpsiphone rufiventer }\end{array}$ & Monarchidae & Passeriformes & - & - & - & 2 & 2 \\
\hline 47. & $\begin{array}{l}\text { Black And White Manikin } \\
\text { Lonchura cucullata }\end{array}$ & Estrildidae & Passeriformes & - & - & 10 & - & 10 \\
\hline
\end{tabular}


Journal of Ecology and Natural Resources

\begin{tabular}{|c|c|c|c|c|c|c|c|c|}
\hline 48. & $\begin{array}{l}\text { Copper Sunbird } \\
\text { Cinnyris cupreus }\end{array}$ & Nectarinidae & Passeriformes & - & - & 12 & - & 12 \\
\hline 49. & $\begin{array}{l}\text { Francolin Double Spur } \\
\text { Pternistis bicalcaratus }\end{array}$ & Phasianidae & Galliformes & - & - & 3 & - & 3 \\
\hline 50. & $\begin{array}{c}\text { Common Bulbul } \\
\text { Pycnonotus barbatus }\end{array}$ & Pycronotidae & Passeriformes & - & - & 4 & 2 & 6 \\
\hline 51. & $\begin{array}{l}\text { Tambourine Dove } \\
\text { Turtur tympanistria }\end{array}$ & Columbidae & Columbiformes & - & - & 10 & - & 10 \\
\hline 52. & $\begin{array}{c}\text { Variable Sunbird } \\
\text { Cinnyris venustus }\end{array}$ & Nectarinidae & Passeriformes & - & - & 15 & - & 15 \\
\hline 53. & $\begin{array}{l}\text { Malachite Sunbird } \\
\text { Nectarinia johnstoni }\end{array}$ & Nectarinidae & Passeriformes & - & - & 2 & - & 2 \\
\hline 54. & $\begin{array}{l}\text { Red-rumped Tinkerbird } \\
\text { Pogoniulus atroflavus }\end{array}$ & Lybiidae & Piciformes & - & - & - & 3 & 3 \\
\hline 55. & African Swift Apus barbatus & Apodidae & Apodiformes & - & - & 8 & - & 8 \\
\hline 56. & $\begin{array}{c}\text { Lizard Buzzard } \\
\text { Kaupifalco monogrammicus }\end{array}$ & Accipitridae & Accipitriformes & - & - & 1 & - & 1 \\
\hline 57. & African Thrush Turdus pelios & Turdidae & Passeriformes & - & - & 6 & - & 6 \\
\hline 58. & $\begin{array}{c}\text { Black Cuckoo Shrike } \\
\text { Campephaga flava }\end{array}$ & Campephagidae & Passeriformes & - & - & 4 & - & 4 \\
\hline 59. & $\begin{array}{l}\text { Red-cheeked Wattle-Eye } \\
\text { Platysteira blissetti }\end{array}$ & Platysteiridae & Passeriformes & - & - & - & 4 & 4 \\
\hline 60. & $\begin{array}{l}\text { Laughing Dove } \\
\text { Streptopelia senegalensis }\end{array}$ & Columbidae & Columbiformes & - & - & 16 & - & 16 \\
\hline 61. & $\begin{array}{c}\text { Mourning Dove } \\
\text { Streptopelia decipiens }\end{array}$ & Columbidae & Columbiformes & - & - & 4 & - & 4 \\
\hline 62. & $\begin{array}{l}\text { Little Greenbul } \\
\text { Eurillas virens }\end{array}$ & Pycnonitidae & Passeriformes & - & - & - & 1 & 1 \\
\hline 63. & $\begin{array}{l}\text { Levaillant's Cuckoo } \\
\text { Clamator levaillantii }\end{array}$ & Cuculidae & Cuculiformes & - & - & - & 1 & 1 \\
\hline 64. & $\begin{array}{c}\text { Palm-nut Vulture } \\
\text { Gypohierax angolensis }\end{array}$ & Accipitridae & Accipitriformes & - & - & - & 1 & 1 \\
\hline 65. & $\begin{array}{c}\text { Red Eye Dove } \\
\text { Streptopelia semitorquata }\end{array}$ & Columbidae & Columbiformes & - & - & 14 & - & 14 \\
\hline 66. & $\begin{array}{l}\text { Pin Tailed Whydah } \\
\text { Vidua macroura }\end{array}$ & Motacillidae & Passeriformes & - & - & 8 & - & 8 \\
\hline 67. & $\begin{array}{l}\text { Little Bee-Eater } \\
\text { Merops pusillus }\end{array}$ & Meropidae & Coraciiformes & - & - & - & 2 & 2 \\
\hline 68. & $\begin{array}{c}\text { Green Hylia } \\
\text { Hylia prasima }\end{array}$ & Sylvioidae & Passeriformes & - & - & - & 1 & 1 \\
\hline 69. & $\begin{array}{c}\text { Splendid Sunbird } \\
\text { Cinnyris coccinigastus } \\
\end{array}$ & Nectarinidae & Passeriformes & 4 & - & 6 & - & 10 \\
\hline 70. & $\begin{array}{l}\text { Western Grey Plaintain Eater } \\
\text { Crinifer piscator }\end{array}$ & Musophagidae & Musophagiformes & - & - & 2 & - & 2 \\
\hline 71. & $\begin{array}{r}\text { Green Headed Negrofinch } \\
\text { Nigrita canicapillus }\end{array}$ & Estrildae & Passeriformes & - & - & - & 1 & 1 \\
\hline
\end{tabular}


Journal of Ecology and Natural Resources

\begin{tabular}{|c|c|c|c|c|c|c|c|c|}
\hline 72. & $\begin{array}{l}\text { African Green Pigeon } \\
\text { Treron calvus }\end{array}$ & Columbidae & Columbiformes & - & - & - & 1 & 1 \\
\hline 73. & $\begin{array}{l}\text { African Grey Parrot } \\
\text { Psittacus erithacus }\end{array}$ & Psittacidae & Psittaciformes & - & 4 & - & - & 3 \\
\hline 74. & $\begin{array}{l}\text { African Pied Hornbill } \\
\text { Lophoceros fasciatus }\end{array}$ & Bucerotidae & Bucerotiformes & - & - & - & 3 & 3 \\
\hline 75. & $\begin{array}{l}\text { Black-casqued Hornbill } \\
\text { Ceratogymna atrata }\end{array}$ & Bucerotidae & Bucerotiformes & - & - & - & 1 & 1 \\
\hline 76. & $\begin{array}{c}\text { Black Winged Oriole } \\
\text { Oriolus nigripennis }\end{array}$ & Oriolidae & Passeriformes & - & - & - & 3 & 3 \\
\hline 77. & $\begin{array}{l}\text { Blue Headed Wood Dove } \\
\text { Turtur brehmeri }\end{array}$ & Columbidae & Columbiformes & - & - & - & 2 & 2 \\
\hline 78. & $\begin{array}{l}\text { Blue Shouldered Robin Chat } \\
\text { Cossypha cyanocamter }\end{array}$ & Muscicapidae & Passeriformes & - & - & - & 1 & 1 \\
\hline 79. & $\begin{array}{l}\text { Blue-Spotted Wood Dove } \\
\text { Turtur afer }\end{array}$ & Columbidae & Columbiformes & - & - & - & 4 & 4 \\
\hline 80. & $\begin{array}{c}\text { Blue Throated Brown Sunbird } \\
\text { Cyanomitra cyanolaema }\end{array}$ & Nectariniidae & Passeriformes & - & - & - & 1 & 1 \\
\hline 81. & $\begin{array}{l}\text { Green Crombec } \\
\text { Sylvietta virens } \\
\end{array}$ & Macrophendae & Passeriformes & - & - & - & 3 & 3 \\
\hline 82. & $\begin{array}{l}\text { Cassin's Flycatcher } \\
\text { Muscicapa cassini }\end{array}$ & Muscicapidae & Passeriformes & - & - & - & 2 & 2 \\
\hline 83. & $\begin{array}{c}\text { Collared Sunbird } \\
\text { Hedydipna collaris }\end{array}$ & Nectariniidae & Passeriformes & - & - & - & 3 & 3 \\
\hline 84. & $\begin{array}{l}\text { White-crested Turaco } \\
\text { Tauraco leucolophus }\end{array}$ & Musophagidae & Musophagiformes & - & - & - & 1 & 1 \\
\hline 85. & $\begin{array}{c}\text { Forest Robin } \\
\text { Stiphrornis erythrothorax }\end{array}$ & Muscicapidae & Passeriformes & - & - & - & 2 & 2 \\
\hline
\end{tabular}

Table 1: Birds Distribution Based on Family, Order and Species on the Land Use Types in Ekiti State.

\begin{tabular}{|c|c|c|c|c|}
\hline Plots & Order & Family & Individual & Species \\
\hline $\mathrm{T} 1$ & 7 & 12 & 86 & 22 \\
\hline $\mathrm{T} 2$ & 11 & 13 & 84 & 24 \\
\hline $\mathrm{T} 3$ & 8 & 15 & 152 & 24 \\
\hline $\mathrm{T} 4$ & 10 & 22 & 70 & 33 \\
\hline
\end{tabular}

Table 2: Table of Order, Family and Species in the Land Use Study Sites in Ekiti State.

\section{Bird Species Abundance in the Four Land Use Types}

A total of 391 individual birds were observed in all the study land use types (Table 2). The abundance of birds varied between the four land use types being highest in gliricidia

plantation (T3) with percentage value $38.87 \%$ of individual birds followed by cashew plantation (T1) which recorded $21.99 \%$, cocoa plantation (T2) $21.48 \%$ while the natural forest (T4) recorded the least percentage value of $17.65 \%$ of individual bird species in the study land use (Table 2). One way ANOVA test for homogeneity was employed to test for significant difference in abundance of bird species between the land uses. The test vividly showed that there were significant differences in the abundance of bird species sighted in the four land use types at $5 \%$ level of significant $(\mathrm{F}=2.09 \mathrm{P}=6.33$ ). Further analysis of the relative abundance of the bird species recorded in the varying land use types indicated that of the bird species, $17,20,12$ and 32 were rare, 4, 4, 7 and 1 were uncommon in the cashew, cocoa, gliricidia plantation and natural forest respectively while 1 and 5 bird species present in the cashew and gliricidia plantation respectively were in frequent category (Figure 1). 


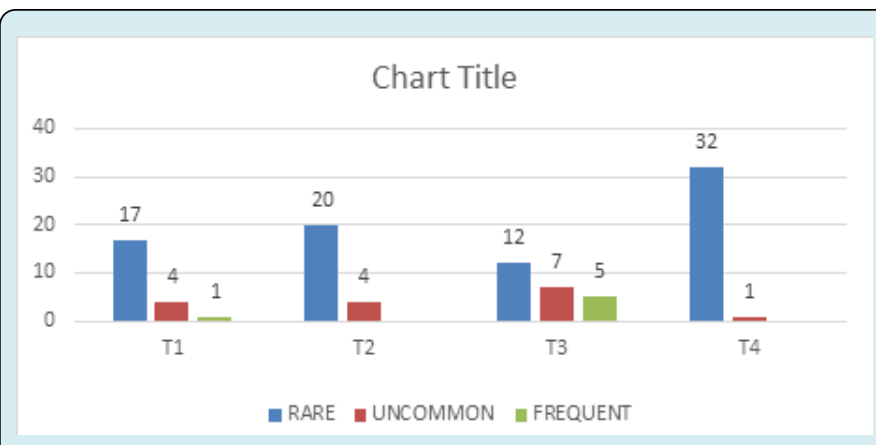

Figure 1: Bird species in different relative abundance categories in the land use types of Ekiti State.

\section{Birds Species Diversity and Community Similarity in Different Land Use Types}

Diversity and evenness analysis was carried out based on the number of individual of bird species in the four (4) land use types and the result vividly showed that there were variations in species diversity between the land use types, natural forest land use type has the highest diversity index followed by cashew plantation then cocoa plantation and lastly gliricidia plantation (Table 3). Even though gliricidia plantation had the highest number of individual birds, it had a relatively low value of diversity. Also the relatively low numbers of individual birds' observable in the natural forest have no retarding influence on the diversity of bird species in the land use type and with regard to species evenness equitability across the study land use types, natural forest registered more even distribution of bird species while cashew plantation had the least even distribution of birds. The variation found in the evenness of bird species in the study sites is an indication that the bird species were unequal in their abundance in the study site. The simpson's index of diversity calculated for each land use types had the highest value in natural forest plantation, indicating that of 100 pairs of bird species observed at random 99 were compose of different species, while cashew plantation land use type recorded the lowest value. The community similarity of birds of the study land use types was low among the study site (Table 4).

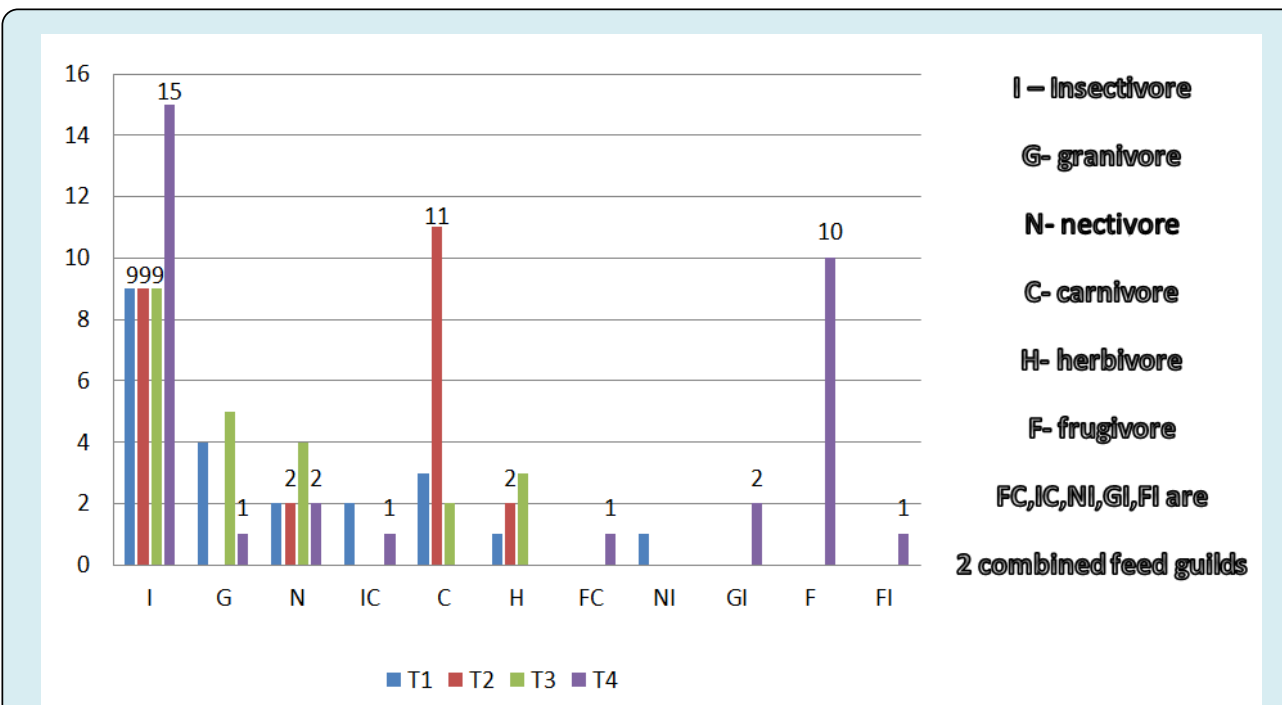

Figure 2: Feeding guilds of bird species in the land use types of Ekiti State.

\begin{tabular}{|c|c|c|c|c|}
\hline Land use types & T1 & T2 & T3 & T4 \\
\hline No of Species & $22^{\mathrm{a}}$ & $24^{\mathrm{a}}$ & $24^{\mathrm{a}}$ & $33^{\mathrm{b}}$ \\
\hline Individuals & $86^{\mathrm{a}}$ & $84^{\mathrm{a}}$ & $152^{\mathrm{b}}$ & $69^{\mathrm{c}}$ \\
\hline Dominance_D & 0.07 & 0.06 & 0.06 & 0.04 \\
\hline Simpson_1-D & 0.93 & 0.94 & 0.94 & 0.96 \\
\hline Shannon_H & 2.88 & 2.97 & 2.91 & 3.34 \\
\hline Evenness_e^H/S & 0.81 & 0.81 & 0.77 & 0.86 \\
\hline Margalef & $4.71^{\mathrm{a}}$ & $5.19^{\mathrm{b}}$ & $4.58^{\mathrm{a}}$ & $7.56^{\mathrm{c}}$ \\
\hline
\end{tabular}

Table 3: Diversity Indices of Bird Species Observed In the Four Land Use Types 


\section{Journal of Ecology and Natural Resources}

\begin{tabular}{|c|c|c|c|c|}
\hline Land use type & T1 & T2 & T3 & T4 \\
\hline T1 & -- & -- & - & - \\
\hline T2 & 0.22 & -- & - & - \\
\hline T3 & 0.17 & 0.13 & - & - \\
\hline T4 & 0.11 & 0.04 & 0.04 & - \\
\hline
\end{tabular}

Table 4: Sorenson's Similarity Indices for the Four Land Use Types.

\section{Avifauna Foraging Structure}

A feeding guild are groups of species that utilize on similar food resources in a habitat and its characterization is mostly based on the type of food being consumed and which invariably determines the feeding behavior of the different bird species and the availability of food resources. In this study eleven types of feeding guild were identified. The entire land use types were dominated by insectivores with $21 \%$ recorded each in cashew, cocoa, gliricidia plantation and $36 \%$ in the natural forest (Figure 2). Red chested cuckoo, Little swift, African pied wagtail, Black cuckoo shrike and Tambourine dove were the most frequent insectivores species inhabiting the study land use types.

\section{Discussion}

The species abundance in this study varies between the four land uses. The highest bird species abundance was recorded in the Gliricidia sepium plantation land use may be due to reasonable food resource availability in this land use type. The nature of arable farming activities existing within and around the Gliricidia sepium plantation may further influence and added to the diverse availability of food for different birds in the habitat. The study conducted by Soka, et al. [26] in Hombolo wetland shows that wetlands provide habitation and aided bird species abundance and richness because they serves as source of food to birds, supports the findings of this study. In regard to the structure of bird communities in the different land use types, birds' diversity is higher in the land uses with high vegetation diversity as occurred in the natural forest. The natural forest examined in this study has mixture of vegetation features, tall large fruit bearing tree species with a wide canopy cover, trees with high diameter at breast height, dead wood stands and the greater diversity of birds in this land use type may be ascribed to these vegetation features and agedness of the habitat. The diverse vegetation flora and features play important roles in the provision of food to birds, support bird nesting, provide good cover for birds hiding place, make available variety of placements for nests, protection against predation and pleasant microclimate for bird species. Soka, et al. [26] asserted that the availability of diverse foods make birds of different feeding guild to dominate an area. Vegetation cover has been reported to have a strong influence on avifauna diversity [27]. The monoculture land use types including the gliricidia plantation present in the farming areas have reasonable bird diversity and richness but most bird species identified in the land uses were generalist with fewer forest dependent species. The overall result of this study shows consistency with the findings of Harvey and Villalobos [13] that agro-monoculture plantations harbor birds that are as abundant species rich and diverse as natural forest habitats. Fjeldsa [28] asserted that habitat disturbance creates increased habitat edge resulting in loss of specialist bird species and immigration of generalist species. The high levels of vegetation alteration as vividly observed in the mono agricultural plantation land uses may invariably be responsible for the heterogeneity in the avian order, families and species composition in the selected four land use types. Elmquist, et al. [29] asserted that nature fragmented system and habitat disturbances lead to loss of specialist species and encourage the generalist species. However, the structure of the monoculture land use types has been distinctly altered which led to the habitation of fewer forest dependent species, so bird diversity is more closely related to the structure and floristic characteristics of land use types. The land use specific relative abundance estimates vividly revealed that 17, 20, 12 and 32 bird species recorded in the Cashew, Cocoa, Gliricidia plantation and Natural forest respectively were in rare birds category and that higher percentages, $77 \%$, 83\% and $96 \%$ of birds recorded in Cashew, Cocoa and the Natural forest respectively, while extremely lower percentages fell into the uncommon and frequent relative abundance categories. The result of the relative abundance category of this study negates the findings of Cody [30] and Igl and Ballard [31] that reported that habitats dominated by woody vegetation or habitats that are structurally and floristically more diverse favours higher relative abundance categories, which was not the case in the present study because none of the bird species recorded were in common or abundance category even in the natural forest considered in this study. The foraging habits of the bird species identified in the selected land use types may explore the variation in flora composition and structural diversity of the land use types, which in accordance with the findings of Pearman [32] that variation in vegetation floristic and structure influences the distribution of bird foraging guilds. Eleven feeding guilds were identified in the study and insectivores were the most dominant group. The insectivorous feeding guild was mostly consisted of bird species from families Cuculidae and Bucerotidae such as Red chested cuckoo (13) and African pied hornbill (3) respectively. In the cashew and gliricidia plantation, the insectivore species were the most abundant species while insectivores' species diversity was found to be highest in the natural forest land use type. This result outcome is fairly in agreement with the discovery of Blake and Loiselle [33] that insectivore's species often 
rank higher in species richness and abundance in tropical forests while Rajaskekara and Verkatesha [34] reported the occurrence of higher numbers of insectivores in agroforests. The availability of a variety of food sources for both adults and young birds within and around the plantation land uses may be responsible for higher frequency of occurrence and abundance of insectivore's species recoded in the study. On the other hand, the insectivores in the cocoa plantation recorded the same value which was obtainable in the other two plantations which is an indication that the frequency of spraying of the farm with insecticide has no pronounced effects on the activities of insects. The next predominant abundant trophic guild group in the land uses was carnivorous birds with African black kite (4), tawny eagle (4) and common kestrel (6) as the most prominent species which were mostly sighted in the cocoa plantation land use where abundance of food sources, such as frogs, snails, squirrels, rats and other small vertebrate species were readily present. Stafford, et al. [35] stated that the abundance of carnivorous bird species in rice fields may be due to the large quantity of food resources, such as polychaetes and mollusks in the rice plots during the migration season. The granivores feeding guild ranked next to carnivores and the granivorous species with higher frequency of occurrence were laughing dove (16), Red-eyed dove (14) and Black and white manikin (10). The nectarivore feeding guild were also more prominent in species richness and abundance in the gliricidia and cashew plantation than in the other land use types, this may be due to the presence of higher flowering intensity in the land use types, the most frequently observable nectarivore bird species were the sunbirds in the family Nectarinidae. Fleming [36] was of opinion that the structure and composition of avian communities changes in space and time with the availability of food resources and that variations are observed to be most visible among birds that feed on patchy and temporary food resources, like nectar and fruit. Cotton [37] found that the abundance and diversity of nectarivores are correlated with an increase in nectar availability. Fewer species of nectarivores inhabits agroecosystems negates the findings of the study. The frugivores were found only in the natural forest land use type and were completely absent in other land uses. The abundance of the available fruit resources may be one of the reasons for the high frugivore bird species richness and abundance in the natural forest. The abundance and richness of fruiting plants is crucial and relates positively with the diversity of frugivorous bird species and foraging behavior in certain habitat types [38]. Finally herbivores were the not abundant in the four land use types and were completely absent in the natural forest. The study provided findings on the effects of land uses on bird species diversity in Ekiti State and the results finding showed that the presence of diverse food sources is highly influential to the diversity and abundance of bird species in the study.

\section{Conclusion}

Birds are critically important in the ecosystem as they act as biological monitors, alerting people about what is happening in an environment. Therefore, it is necessary to have a data base for the land use effects on bird species diversity in Ekiti State. The result of this study revealed the variation in the diversity, abundance and distribution of birds in the study land uses and their most preferred land use type. The birds found were mostly insectivorous species and have the highest species composition and abundance in the natural forest and gliricidia plantation respectively. The relative abundance of birds encountered during the study fell mostly on rare category. The fact that more species of bird were not observed does not indicate that birds were totally absent in the study land use but it is an indication that the families of birds were also rare in the land use types and express the urgent need to map out effective strategy for the conservation of birds in the land use types of Ekiti State.

\section{References}

1. Deikumah JP, McAlpine CA, Maron M (2014) Mining matrix effects on West African rainforest birds. Biological Conservation 169: 334-343.

2. Kennedy CM, Marra PP, Fagan WF, Neel MC (2010) Landscape matrix and species traits mediate responses of Neotropical resident birds to forest fragmentation in Jamaica. Ecological Monographs 80(4): 651-669.

3. Ruiz-Guerra B, Renton K, Dirzo R (2012) Consequences of fragmentation of tropical moist forest for birds and their role in predation of herbivorous insects. Biotropica 44(2): 228-236.

4. Raman TRS (2003) Assessment of census techniques for interspecific comparisons of tropical rainforest bird densities: A field evaluation in the Western Ghats, India. Ibis 145(1): 9-21.

5. Naidoo R (2004) Species richness and community composition of songbirds in tropical forest agricultural landscape. Animal Conservation 7(1): 93-105.

6. Chamberlain DE, Fuller RJ, Brunce RGH, Shrubb M (2000) Changes in the abundance of farmland birds in relation to the timing of agricultural intensification in England and Wales. Journal of Applied Ecology 37(5): 771-788.

7. Benton TG, Bryant DM, Cole L, Crick HQP (2002) Linking agricultural practices to insect and bird populations: a historical study over three decades. Journal of Applied Ecology 39(4): 673-687. 
8. Cline BB, Hunter ML (2016) Movement in the matrix: Substrates and distance-to-forest edge affect post metamorphic movements of a forest amphibian. Ecosphere 7(2): 11991-1202.

9. Siebert SF (2002) From Shade to Sun -grown perennial crops in Sulawesi, Indonesia. Implications for biodiversity conservation and soil fertility. Biodiversity and Conservation 11: 1889-1902.

10. Philpott SM, Arendt WJ, Armbrecht I, Bichier P, Diestch TV, et al. (2008) Biodiversity loss in Latin American Coffee landscapes: Review of the evidence on ants, birds and trees. Conservation Biology 22(5): 1093-1105.

11. Wood A, Stedma EP, Mang J (2000) Ten case study an overview. In: Wood (Ed.), The Root Causes of Biodiversity Loss. et al.). Earth scans Publication Ltd, UK, pp: 36-37.

12. Christopher PW (2003) The Implication of Land Use Change on Forestry Biodiversity. A case study of the "Half Mile Strip" on the Mount Kilimanjaro, Tanzania. LUCID. Project working paper 30. Nairobi. Kenya. International Livestock research Institute.

13. Harvey CA, Villalobos JAG (2007) Agroforestry systems conserve species-rich but modified assemblages of tropical birds and bats. Biodiversity and Conservation 16(8): 2257-2292.

14. Rodrigues JC, Pellizari VH, Mueller R, Baek K, Jesus EDC, et al. (2013) Conservation of the Amazon rainforest to agriculture results in biotic homogenization of soil bacterial communities. Proceedings of the National Academy of Sciences 110(3): 988-993.

15. Schroth G (2004) Agroforestry and Biodiversity conservation in tropical landscapes. Washington, D.C., Island Pess.

16. Norris K (2008) Agriculture biodiversity conservation opportunity knocks. Conservation Letters 1(1): 2-11.

17. Perfecto I, Vandermeer J (2002) Quality of agroecological matrix in a tropical montane landscape: Ants in Coffee plantations in Southern Mexico. Conservation Biology 16(1): 174-182.

18. Harvey CA, Medina A, Sanchez DM, Maes JC, Casanoves F, et al. (2006) Pattern of animal diversity in different forms of tree cover in agricultural landscape. Ecological Applications 16(5): 1986-1999.

19. Fahrig L, Girard J, Duro D, Pasher J, Smith A, et al. (2015) Farmlands with smaller crop fields have higher within field biodiversity. Agriculture Ecosystems and Environment 200: 219-234.
20. Bolwig S, Pomeroy D, Tushabe H, Mushabe D (2006) Crops, trees, and birds: Biodiversity change under agricultural intensification in Uganda's farmed landscapes. Danish Journal of Geography 106(2): 115-130.

21. Drakenberg O (2007) Environmental Policy Brief for the Lake Victoria Basin. School of Economics and Commercial Law. Goteborg University 36: 2-36.

22. Bibby CJ, Burgess ND, Hill DA, Mustoe SH (2000) Bird Census technique. Academic Press, London.

23. Bibby CJ, Jones M, Marsden S (1998) Expendition Field Techniques: Bird Surveys. Expendition Advisory Centre. Royal Geographical Society, London.

24. Oksanen L (2001) Logic of experiments in ecology: Is pseudo- replication a pseudo-issue? Oikos 94:27-38.

25. Borrow N, Demey R (2004) Birds of western Africa, published by Christopher helm an imprint A and C Black publishers Ltd, 37 W Soho square, London WSD-3QZ.

26. Soka GE, Munishi PK, Mgina B (2013) Species diversity and abundance of Avifauna in and around Hombolo Wetland in Central Tanzania. International Journal of Biodiversity and Conservation 5(11): 782-790.

27. Radford JQ, BennettAF, Cheers GJ (2005) Landscape-level thresholds of habitat cover for woodland-dependent birds. Biological Conservation 124(3): 317-337.

28. Fjeldså J (1999) The impact of human forest disturbance on the endemic avifauna of the Udzungwa Mountains, Tanzania. Bird Conservation International 9(1): 47-62.

29. Elmquist A, Langel Ü (2003) In vitro uptake and stability study of pVEC and its all-D analog. Biological Chemistry 384(3): 387-393.

30. Cody ML (1985) Habitat selection in birds. Academic Press.

31. Igl LD, Ballard BM (1999) Habitat associations of migrating and overwintering grassland birds in southern Texas. The Condor 101(4): 771-782.

32. Pearman PB (2002) The scale of community structure: habitat variation and avian guilds in tropical forest understory. Ecological Monographs 72(1): 19-39.

33. Blake JG, Loiselle BA (2001) Bird assemblages in secondgrowth and old-growth forests, Costa Rica: perspectives from mist nets and point counts. The Auk 118(2): 304326.

34. Rajashekara S, Venkatesha MG (2014) Insectivorous 
bird communities of diverse agro-ecosystems in the Bengaluru region, India. Journal of Entomology and Zoology Studies 2(5): 142-155.

35. Stafford JD, Kaminski RM, Reinecke KJ (2010) Avian foods, foraging and habitat conservation in world rice fields. Water birds 33(1): 133-150.

36. Fleming TH (1992) How do fruit and nectar feeding birds and mammals track their food resources? In: Hunter
MD, Ohgushi T, Prick PW (Eds.), Effects of resource distribution on animal- plant interactions. Academic, San Diego, pp: 355- 391.

37. Cotton PA (2006) Seasonal resource tracking by Amazonian hummingbirds. Ibis 149(1): 135-142.

38. Moegenburg S, Levey DJ (2003) Do frugivores respond to fruit harvest? An experimental study of short-term responses. Ecology 84(10): 2600-2612. 